\title{
Temperate Bacteriophages for Streptomyces coelicolor A3(2) Isolated from Soil
}

\author{
By J. E. DOWDING* AND D. A. HOPWOOD \\ John Innes Institute, Colney Lane, Norwich NOR $70 \mathrm{~F}$
}

(Received 25 April 1973)

\begin{abstract}
SUMMARY
Three phages, isolated from soil samples collected in different localities, produced turbid plaques with a characteristic morphology on Streptomyces coelicolor A3(2). Surviving growth from the plaques was lysogenic: when isolated and purified it was resistant to lysis by the homologous phage and released phage at a low frequency during growth. The three phages were homoimmune. The chromosomal attachment site of one of the prophages, $\mathrm{VP}_{5}$, was mapped in a position between hisE and cysD - that is, in a region devoid of standard markers - on the circular linkage map of the host, by crossing defective lysogens with sensitive strains. The host range of $\mathrm{VP}_{5}$ was restricted; only certain strains belonging to the $S$. coelicolor $(S$. violaceoruber) group were attacked. VP5 gave rise to clear-plaque mutants, incapable of lysogenizing the host, at a spontaneous frequency of $\mathrm{IO}^{-4}$, which was increased by u.v. irradiation and by hydroxylamine mutagenesis; the clear-plaque mutants were incapable of lysing strains carrying the wild-type prophage. Electron microscopy showed that VP 5 belongs to group B of Bradley's morphological classification.
\end{abstract}

\section{INTRODUCTION}

Welsch (1956) was the first to show, by rigorous criteria, the occurrence of true lysogeny in members of the genus Streptomyces. Welsch (1959) was unable to demonstrate lysogenization by any phages isolated from natural sources; instead, spontaneously occurring lysogenic cultures were shown to exist when they released phages capable of forming plaques on sensitive indicator strains. The same experience was reported in later Russian publications (see references in Lomovskaya, Mkrtumian, Gostimskaya \& Danilenko, 1972). The most detailed of these studies are those of Lomovskaya, Emeljanova \& Alikhanian (I97I) and Lomovskaya et al. (1972), who showed that Streptomyces coelicolor A3(2), the strain in which most genetical work has been done (Hopwood, 1967; Sermonti, I969; Hopwood, Chater, Dowding \& Vivian, I973), harbours a prophage, presumed to be defective because of its very low spontaneous frequency of induction. Once released, the phage formed large turbid plaques on a sensitive indicator strain. The phage isolated from these plaques, called $\varnothing_{3} \mathrm{C}_{3} \mathrm{I}$, readily lysogenized the indicator strain, and also $\mathrm{A} 3(2)$ and its derivatives provided that these had been cured of the defective prophage; all the artificially prepared lysogens then released phage at an easily detectable frequency. A chromosomal attachment site was demonstrated by crossing lysogenic and non-lysogenic derivatives of $\mathrm{A3}(2)$.

In a study in which 28 phages capable of forming plaques on Streptomyces coelicolor A3(2) were isolated from soil samples, Dowding (1972) found that some produced turbid plaques from which lysogenic growth could be isolated. One, VP5, is described in the present

* Present address: Department of Biochemistry, University of Wisconsin, Madison, Wisconsin 53706, U.S.A. 
paper. This is, as far as we are aware, the first published report of a temperate phage for an actinomycete being first detected as free virions, rather than as a prophage harboured by a lysogenic host.

\section{METHODS}

Bacterial strains. These were Streptomyces coelicolor A3(2) (Hopwood, 1959) and its mutant and recombinant derivatives, and strain 66 (our stock no. 1326) kindly supplied by Dr N. D. Lomovskaya, Institute of Genetics and Selection of Industrial Micro-organisms, Moscow, U.S.S.R.

General bacterial techniques. Complete (CM) and minimal (MM) media and standard cultural techniques were those described by Hopwood (1967). All incubations were at $30^{\circ} \mathrm{C}$.

Genetic analysis and host mutagenesis. Haploid recombinant selection was done in the way described by Hopwood (1967). Non-selective analysis was performed by the method of Hopwood, Harold, Vivian \& Ferguson (1969). Mutagenesis by $N$-methyl- $N$-nitro- $N$ nitrosoguanidine (NTG) was by the method of Delić, Hopwood \& Friend (1970) using $3 \mathrm{mg} / \mathrm{ml}$ in $0.05 \mathrm{M}$-tris-maleic acid buffer, $\mathrm{pH} \mathrm{9,} \mathrm{for} \mathrm{I} \mathrm{h}$.

Phage isolation. VP5 was isolated by the method of Welsch, Minon \& Schönfeld (1955) from a soil sample from Norwich, Norfolk. The method of specific enrichment (Dowding, I973) on Streptomyces coelicolor A3(2) was used to isolate VP7 and VPI4 from soil samples collected from Wymondham and Old Buckenham, Norfolk, respectively.

General phage techniques. The methods and media used in phage propagation and assay were those described by Dowding (1973). It was found that VP5 did not reproduce under the shaking-flask lysate conditions described for the virulent phage VPII, so that the confluent-plate lysate method was used exclusively, yielding lysates containing about $10^{8}$ plaque-forming units $/ \mathrm{ml}$.

Phage mutagenesis. Ultraviolet irradiation was performed as described by Dowding (1973). For chemical mutagenesis, hydroxylamine hydrochloride (B.D.H. Chemicals Ltd, Poole, Dorset) in distilled water at a concentration of $0.4 \mathrm{M}$ was brought to $\mathrm{pH} 6.8$ with a small volume of $6 \mathrm{~N}-\mathrm{NaOH}$. Equal volumes of this solution and of VP5 lysate were mixed and incubated at $30{ }^{\circ} \mathrm{C}$. Samples were withdrawn at intervals, immediately diluted at least I000-fold, and plated.

Electron microscopy. Phage from lysates was concentrated, partially purified and examined by the methods described by Dowding (1973).

\section{RESULTS}

\section{Plaque morphology of VP5}

The plaque morphology of VP5 after overnight incubation is shown in Fig. 1 ; the plaques have a clear centre surrounded by a broad turbid halo. On continued incubation the plaques of VP5, unlike those of the virulent phage VPII (Dowding, 1973), increased in size and changed in morphology. After 3 days' incubation (Fig. 2) the turbid halo seen in young plaques (Fig. I) became converted into a zone of vigorous bacterial growth, surrounded by a second zone of lysis, and this in turn was surrounded by a turbid halo. Two other phages, VP7 and VPI4, showed plaque morphologies identical to that of VP5.

\section{Isolation and characterization of lysogens}

Since a clear zone of lysis was present outside the surviving bacterial growth in each mature plaque of $\mathrm{VP}_{5}$, it was unlikely that host survival was due to the onset, with age, of 


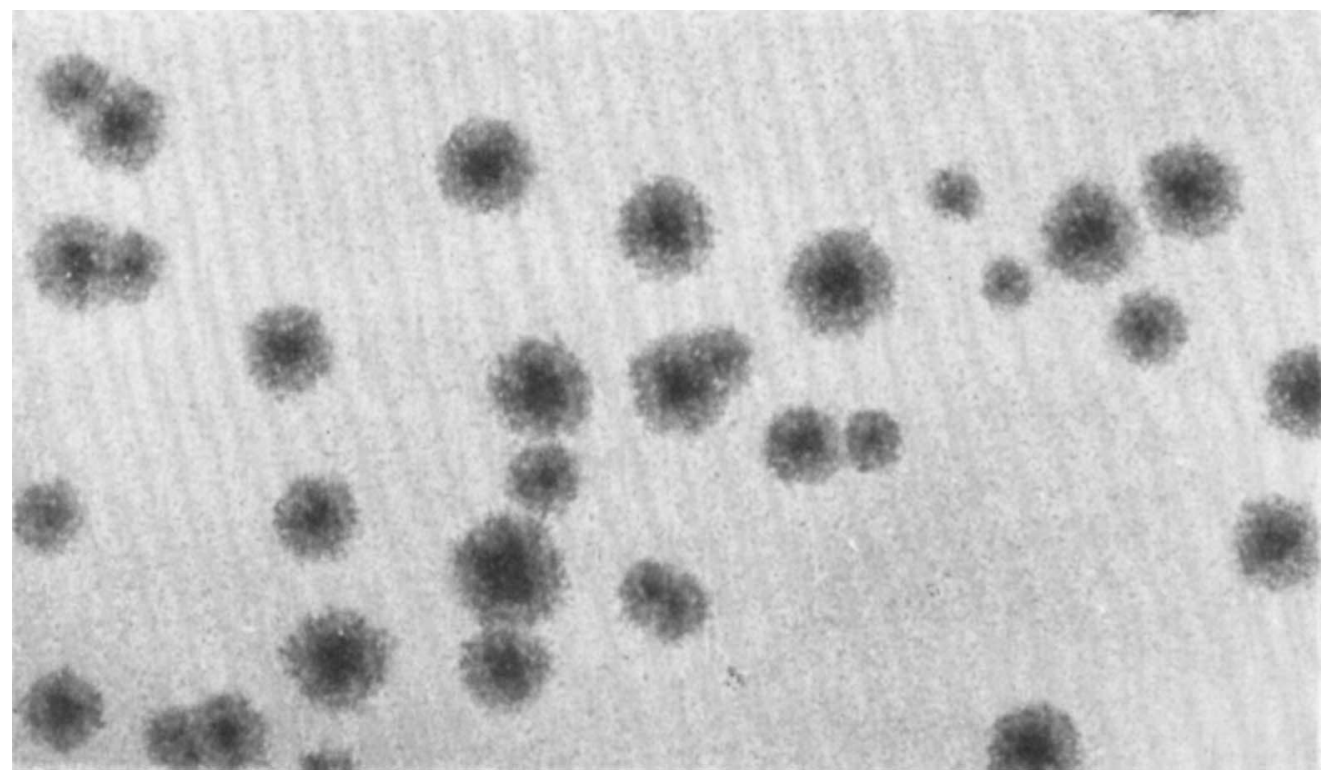

Fig. I Plaques of VP5 on Streptomyces coelicolor A3(2) after overnight incubation $(\times 4)$.

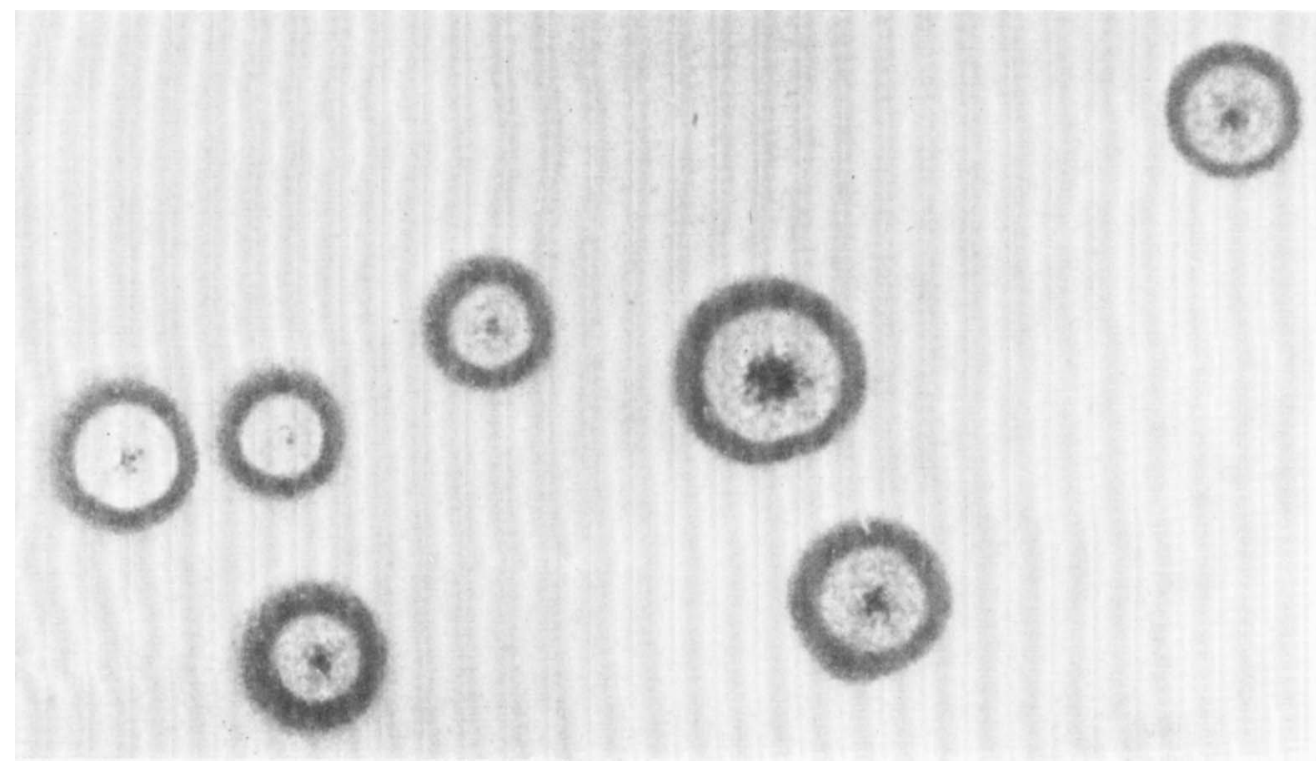

Fig. 2. Plaques of $\mathrm{VP}_{5}$ on Streptomyces coelicolor $\mathrm{A}_{3}(2)$ after incubation for 3 days $(\times 5)$.

physiological incompetence to support phage reproduction. To test the hypothesis that resistance was due to lysogenization, the resistant growth from ten plaques grown on strain A200 (ade-vIO pheAI strAI UF) was streaked on MM supplemented with adenine and phenylalanine. When the colonies had sporulated, a number were purified by further streakings and tested for resistance to VP5. All colonies tested were resistant and retained the genetic markers of the starting strain. One colony, strain 1829 , was chosen for further study. 


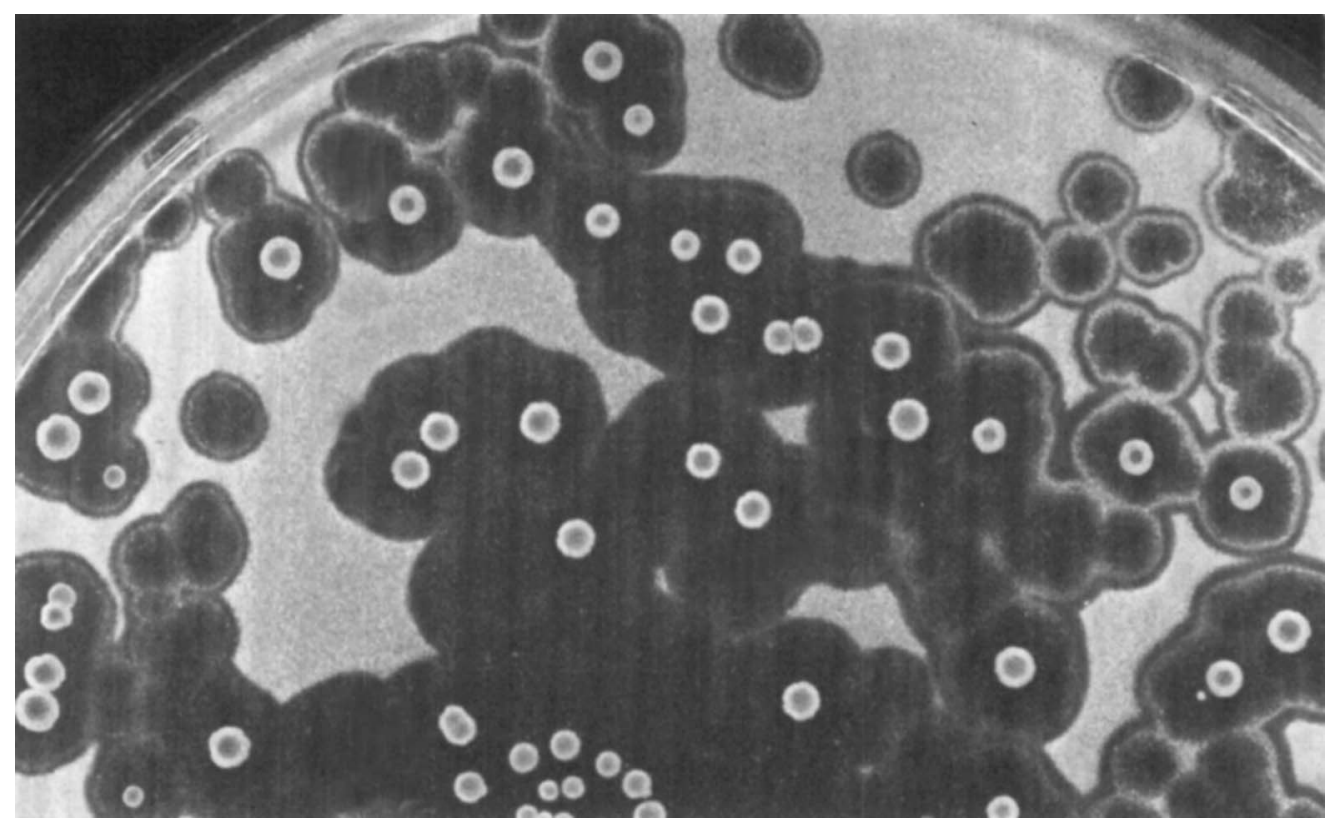

Fig. 3. Colony-centred plaques obtained by plating strain I 829, lysogenic for VP5, on nutrient agar and overlaying with the phage-sensitive parent strain, A200, after 3 days' incubation. The figure shows the culture after a further $24 \mathrm{~h}$ incubation. Note plaques caused by free phages dispersed from the colonies during the overlaying procedure $(\times 2 \cdot 2)$.

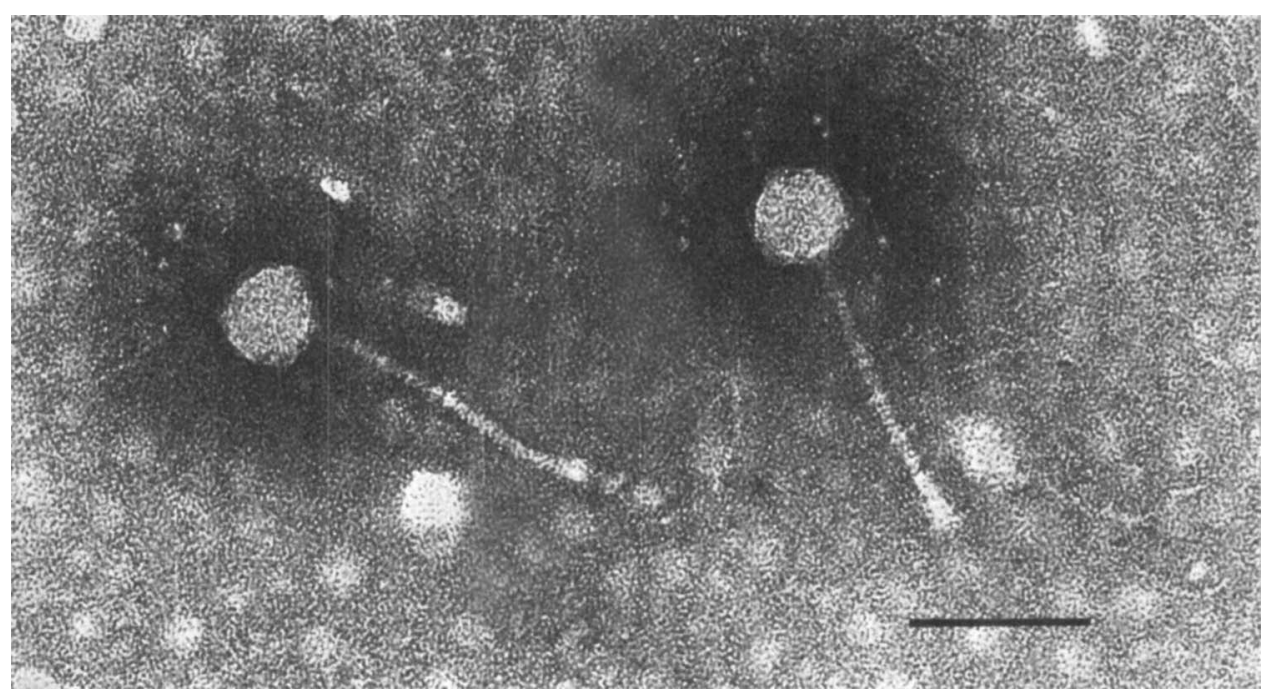

Fig. 4. Electron micrograph of two particles of VP5 negatively stained with neutral potassium phosphotungstate. The marker bar represents $100 \mathrm{~nm}$.

To test for phage release, a spore suspension of strain 1829 was prepared and washed three times by centrifugation and resuspension in distilled water. The final wash was membrane-filtered and plated on A200 to assay free phage. Suitable dilutions of the washed spore suspension were plated and immediately overlaid with A200 to test for spontaneous phage 
release. The plaque counts from these platings are shown in Table $\mathrm{I}$. The most striking observation was that the counts from the 1829 platings increased gradually on prolonged incubation. Very small new plaques appeared and were observed to grow in diameter. No such increase in plaque count was seen on the plates on which free phage from the washings had been spread and indeed has never been observed when free VP5 has been assayed. The I 829 suspension used for plating contained $\mathrm{I} \times 1 \mathrm{IO}^{7}$ colony forming units $/ \mathrm{ml}$. Evidently 3 to $4 \%$ of the colonies derived from the spores plated had released phages at some time earlier than $64 \mathrm{~h}$ under these conditions.

Further plates of the washed 1829 spore suspension were overlaid with A200 after 3 days' growth and incubated overnight. Every colony was surrounded by a zone of phage lysis (Fig. 3). Control colonies of A200 similarly overlaid showed no signs of lysis.

These observations provided evidence that $\mathrm{VP}_{5}$ could readily lysogenize derivatives of Streptomyces coelicolor A3(2), and the lysogens slowly released phage during growth. Such phage release by presumptive lysogens has invariably been detected when it has been sought even after many single colony isolations, some of them on $\mathrm{CM}$, which does not allow plaque formation by this or some other actinophages (Dowding, 1972). The spontaneous frequency of induction could not be increased by u.v. irradiation.

\section{Similar wild-type phages}

Presumptive lysogens carrying prophages of the two other phages with VP5-like plaque morphology, VP7 and VPI4, were prepared. A lysogen carrying any one of the three prophages showed no signs of lysis by any of the three phages. Thus the three phages appeared to be homoimmune.

\section{Clear-plaque mutants}

Occasional clear-plaque mutants were observed on plates sown with large numbers of VP5 particles. The spontaneous frequency was determined by picking five well-isolated single plaques of wild-type VP5 into $2 \mathrm{ml}$ broth samples with sterile Pasteur pipettes, allowing the phages to soak out for $\mathrm{I}$ to $2 \mathrm{~h}$, and membrane filtering the resulting phage suspensions. These were plated, and the plates were examined for clear and sectored plaques after incubation for $24 \mathrm{~h}$. All presumptive mutants were picked and retested. Out of about 12000 plaques from each of the five platings the numbers of clear-plaque mutants were: $0, \mathrm{I}, \mathrm{I}, \mathrm{I}, 3$, giving a spontaneous mutant frequency of about $\mathrm{I} \times \mathrm{IO}^{-4}$.

After u.v. irradiation to about I \% survival, nine clear-plaque mutants were found among 4I $8 \mathrm{I}$ plaques $\left(2 \times \mathrm{IO}^{-3}\right)$. Treatment with $0.2 \mathrm{M}$-hydroxylamine induced significant proportions of clear-plaque mutants, up to $6 \times 10^{-3}$ at $1 \%$ survival (Table 2 ).

It was found that strains lysogenized with wild-type VP5 were resistant to lysis by clearplaque mutants.

\section{A chromosomal location for the VP5 prophage}

Since a possible complication in attempts to map the prophage attachment site by looking for segregation of phage resistance versus sensitivity in crosses of lysogenic with nonlysogenic strains was zygotic induction (Jacob \& Wollman, 1956), a UF strain was chosen as the lysogenic parent. In crosses with NF donors, UF strains act as recipients (Hopwood et al. 1969). The lysogen, strain I829 (ade-vIo pheAI strAI) was crossed with NF strain I 258 (proAI hisAI $\arg A I$ cysDI8 uraAI strAI), and a sample of 150 progeny recovered on non-selective medium was tested for standard markers and for sensitivity or resistance to $\mathrm{VP}_{5}$ by replica plating to nutrient agar spread with a clear-plaque mutant of VP5. All the colonies were $\mathrm{VP}_{5}$-resistant. Owing to the nature of the zygotes in $\mathrm{NF} \times \mathrm{UF}$ crosses (Hopwood 
Table I. Release of VP5 by lysogenic strain 1829

$\begin{array}{ccc}\text { Incubation time (h) } & \overbrace{\text { Final wash filtrates }}^{\text {No. of plaques per ml of suspension plated }} & \text { Washed spore suspension } \\ 18 & \mathrm{I} \cdot 4 \times \mathrm{IO}^{2} & \mathrm{I} \cdot 8 \times 1 \mathrm{IO}^{5} \\ 40 & \mathrm{I} \cdot 4 \times \mathrm{IO}^{2} & 3.0 \times 10^{5} \\ 64 & \mathrm{I} \cdot 4 \times \mathrm{IO}^{2} & 3.6 \times 10^{5}\end{array}$

Table 2. Induction of clear-plaque mutants during inactivation of VP 5 by 0.2 M-hydroxylamine

$\begin{array}{ccccc}\begin{array}{c}\text { Time of } \\ \text { treatment }(\mathrm{h})\end{array} & \begin{array}{c}\text { Survival } \\ (\%)\end{array} & \begin{array}{c}\text { No. of plaques } \\ \text { examined }\end{array} & \begin{array}{c}\text { No. of clear } \\ \text { plaques }\end{array} & \begin{array}{c}\text { Clear plaques } \\ \text { per } \text { I0 }^{3} \text { survivors }\end{array} \\ 0 & 100 & 3989 & 0 & - \\ \text { I } & 99 & 2314 & \text { I } & 0.4 \\ 2 & 69 & 1702 & \text { I } & 0.6 \\ 4 & 33 & 2184 & 3 & 1.4 \\ 6 & 7 & 1716 & 6 & 3.5 \\ 8 & \text { I } & 2898 & 17 & 5.9\end{array}$

et al. 1969), this result was compatible with chromosomal location in the right-hand half of the linkage map, since for markers in this region the frequency of the allele coming from the UF parent approaches $100 \%$. However, phage release by the lysogenic parent in the cross, followed by lysogenization or lysis of sensitive individuals, could have resulted in all progeny becoming lysogenic, irrespective of any chromosomal location of the prophage. This complication was avoided by isolating a defective lysogen, as follows. Strain I829 was treated with NTG and the surviving colonies on nutrient agar were overlaid with A3(2) to indicate phage release. Out of $335^{8}$ colonies tested, one was found not to release phage. Moreover, on testing it was found to retain $\mathrm{VP}_{5}$-resistance, and this colony (strain 1854) was selected as presumptively carrying a defective $\mathrm{VP}_{5}$ prophage.

In a cross of UF strain I 854 with NF strain 949 ( $\arg A$ I proAI cysAI 5 nicAI uraAI), a sample of 100 progeny were all VP5-resistant. One of these recombinant progeny, NF strain 1860 ( $\arg$ AI proAI cysAI 5 nicAI uraAI pheAI strAI), was crossed with a non-lysogenic UF strain, I5I6 (his $C 9$ ), and this time all roo progeny tested were $\mathrm{VP}_{5}$-sensitive. Both results were compatible with a chromosomal location in the right-hand half of the map, and this was revealed by a further cross of two UF strains (UF $\times$ UF crosses have a low but reproducible fertility, see Vivian \& Hopwood, I970). The progeny from this cross showed an unambiguous segregation of $\mathrm{VP}_{5}$-resistance versus sensitivity, leading to the identification of a map location between $\arg A$ and $\operatorname{cys} D$.

Lomovskaya et al. (197I) identified a chromosomal location for the defective prophage of $\varnothing C_{3} \mathrm{I}$, also between $\arg A$ and $\operatorname{cys}_{s} D$; this was done in crosses of strains carrying the wildtype defective prophage with strains cured of the prophage. $\varnothing_{3} \mathrm{C}_{3}$ and $\mathrm{VP}_{5}$ are heteroimmune, as shown by the sensitivity of $\mathrm{A} 3(2)$ and its derivatives (which carry the defective ØC3 1 prophage) to $\mathrm{VP}_{5}$, and by our finding that $\mathrm{I}_{32} 6$ lysogenized by either of the two phages remained susceptible to the other. To see whether the attachment sites of VP5 and ØC 3 I were closely linked a cross was performed between a defective VP5 lysogen, also carrying the wild-type defective $\varnothing_{3}$ I prophage, and a strain that had been cured of the defective $\varnothing_{3} I$ prophage (and not carrying VP5); this strain was isolated from the survivors of u.v. irradiation to a survival of $10^{-4}$ as described by Lomovskaya et al. (1971). Progeny from the cross were classified in respect of resistance versus sensitivity to each of the two phages by 
Table 3. Mapping of the VP5 prophage attachment site

Strain 1862 (argAI proAI uraAr pheAI VP5 $5^{\mathrm{r}} \varnothing \mathrm{C}_{3} \mathrm{I}^{\mathrm{r}}$ : outer circle) was crossed with strain 1905 (hisAI leuB5 $\mathrm{VP}_{5}^{\mathrm{B}} Ø \mathrm{C}_{3 \mathrm{I}^{\mathrm{B}}}$ : inner circle) and recombinants inheriting his ${ }^{+}$and phe $^{+}$(indicated by triangles) were selected and classified in respect of non-selected auxotrophic markers and phage resistance versus sensitivity. Numbers represent allele frequencies amongst a sample of the selected progeny. (Segregation of proAI and $u r a A I$, in the left-hand half of the map, has been ignored.)

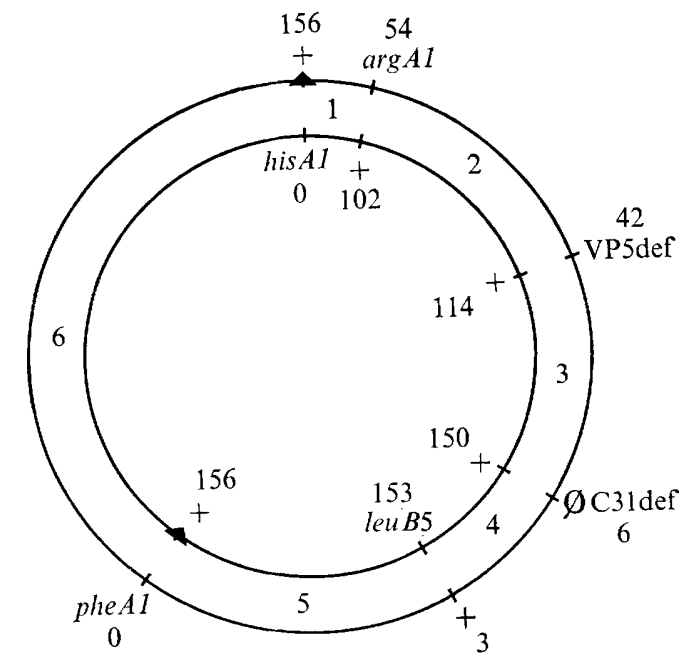

\begin{tabular}{|c|c|}
\hline Genotype* & Crossovers in intervals \\
\hline leuB5 & I, 6 \\
\hline $\arg A I \operatorname{leu} B 5$ & 2,6 \\
\hline $\arg A I$ leuB5 VP5def & 3,6 \\
\hline $\arg A I$ leuB5 VP5def $\varnothing \mathrm{C}_{3} \mathrm{Idef}$ & 4,6 \\
\hline $\arg A I$ VP5 def $\varnothing C_{3} I$ def & 5,6 \\
\hline leuB5 VP5def & $I, 2,3,6$ \\
\hline leuB5 VP5def $\varnothing C_{3}$ Idef & $I, 2,4,6$ \\
\hline $\arg A I \varnothing C_{3} I \operatorname{def}$ & $2,3,5,6$ \\
\hline
\end{tabular}

* Wild-type alleles of auxotrophic markers, and phage sensitivity, omitted.

replication to plates spread with clear-plaque phage mutants. The results (Table 3 ) confirmed the location of the defective $\varnothing_{3}$ I prophage anti-clockwise of leuB and showed the $\mathrm{VP}_{5}$ attachment site to be between $\arg A$ and the attachment site of $\varnothing \mathrm{C}_{3} \mathrm{I}$, and not close to the latter.

There are several other markers between $\arg A$ and the beginning of the 'silent region' that occupies nearly a quarter of the linkage map between $\arg A$ and $\operatorname{cys} D(\operatorname{Hopwood}, 1966 a, b)$; the locus nearest to the silent region is hisE. In a further cross (Table 4) between a recombinant carrying the defective VP5 prophage and a sensitive strain, the attachment site of VP5 was found to be clockwise of hisE, that is within the 'silent region'.

\section{Host range of $V P_{5}$}

The host range of many bacteriophages attacking actinomycetes is wide; the virulent phage VPI I, isolated on Streptomyces coelicolor A3(2), had a remarkably wide host range (Dowding, 1973). VP5 and a sample of its clear-plaque mutants were tested on a collection of Streptomyces wild-types, including those used by Dowding (1972) for VPII. Negative 
Table 4. Mapping of the VP5 prophage attachment site

See Table 3 for procedure (hisE6 was classified by means of its alternative requirement for adenine).

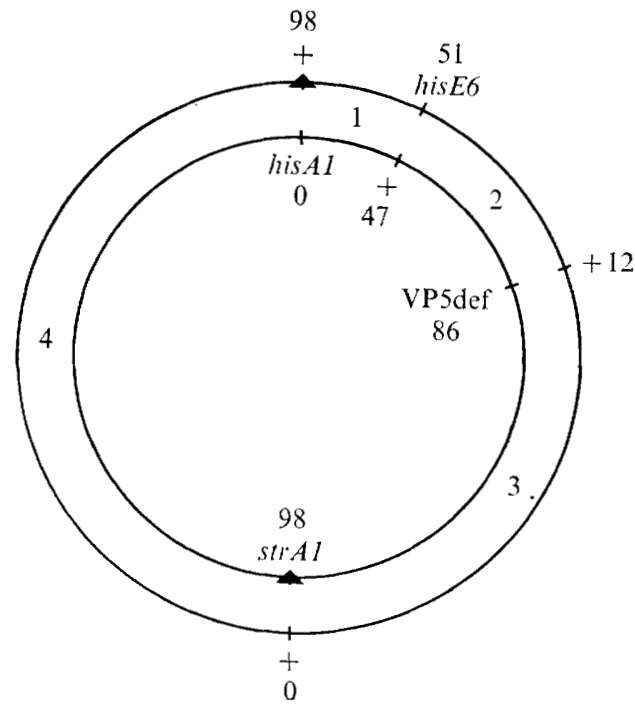

\begin{tabular}{lcc}
\multicolumn{1}{c}{ Genotype* } & Crossovers in intervals & No. \\
VP5def & I, 4 & 46 \\
hisE6 VP5def & 2,4 & 40 \\
hisE6 & 3,4 & I I \\
\multicolumn{1}{c}{ - } & I, 2, 3, 4 & I
\end{tabular}

* Wild-type alleles of auxotrophic markers, and phage sensitivity, omitted.

results were obtained in all cases except on three $S$. coelicolor $(S$. violaceoruber $)$ wild-types and strain 1326; one other $S$. coelicolor ( $S$. violaceoruber) wild-type was insensitive. Thus the host-range of the temperate $\mathrm{VP}_{5}$ is much narrower than that of virulent VPII.

\section{Morphology of $V P_{5}$}

Figure 4 shows two particles of VP5 negatively stained with neutral potassium phosphotungstate. The phage is seen to have a hexagonal head and a long, flexible striated tail. It thus belonged to group B of Bradley's (1967) morphological classification. Measurements taken from 20 particles showed the dimensions to be as follows: head length, $58 \cdot 6 \pm \mathrm{I} \cdot 2 \mathrm{~nm}$; head width, $58.4 \pm \mathrm{r} \cdot 5 \mathrm{~nm}$; tail length $\mathrm{I} 66 \pm 2.5 \mathrm{~nm}$; tail width between Io and $\mathrm{I} 2 \mathrm{~nm}$. VP7, one of the other independently isolated phages homoimmune with VP5, had the same morphology and dimensions as $\mathrm{VP}_{5}$.

\section{DISCUSSION}

The majority of reports on lysogeny in Streptomyces have been concerned with attempts to isolate viruses by the induction of prophages which might be naturally present in the strains tested. The only published attempts to lysogenize a culture with phage not produced by it appear to be those of Welsch (1959). One of the main advantages of lysogenizing a strain with such a phage is that an indicator culture does not have to be discovered (see Shirling, 


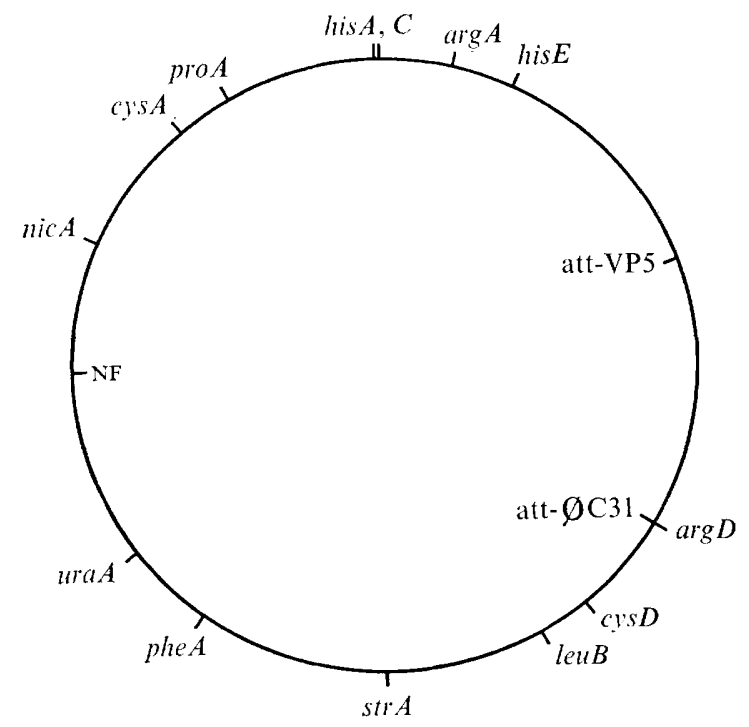

Fig. 5. Linkage map of Streptomyces coelicolor A3(2). On the outside of the circle are the standard loci referred to in this paper. On the inside of the circle are the attachment sites of phages $\varnothing_{3} I$ (Lomovskaya et al. I97I) and VP5 (data in the present paper). The point NF is the site of interaction of plasmid SCPI (Vivian, I97I) with the chromosome in the NF fertility type (Vivian \& Hopwood, 1970; Hopwood et al. 1973).

I956). Welsch was unable to show, for a Streptomyces griseus strain, that any of the survivors of phage infection were true lysogens. He discussed the occurrence of 'pseudolysogeny', which is common in streptomycetes and probably arises from their mycelial growth habit. This results in a culture containing cells of varying morphological and physiological states, not all favourable to phage propagation. Thus a culture originally infected with a virulent phage may come to contain considerable numbers of phenotypically resistant but genotypically sensitive units. Unless the culture is stringently cloned on subculture, ideally under conditions unfavourable to phage adsorption, it may continue to show phage 'release' indefinitely, even though it contains no truly lysogenic units.

Evidence described above indicates that three phages isolated independently from soil samples collected from sites separated by at least $10 \mathrm{~km}$ are able to lysogenize Streptomyces coelicolor $\mathrm{A} 3(2)$. The three main pieces of evidence are: the surviving growth in the plaques was, when isolated and purified, resistant to the phage; the resistant strains, after exhaustive purification, continued to release phage spontaneously, giving rise to colony-centred plaques on a lawn of the sensitive parent strain; and a chromosomal attachment site for the presumed prophage was recognized.

The rise in plaque counts observed when spores of a lysogen were plated with a sensitive strain (Table I) may be explained by the reasonable assumption that lysogenic colonies had to reach a critical size before there was a high statistical chance of at least one of their constituent cells releasing phage by spontaneous induction. Only 3 to $4 \%$ of the spores plated appeared to give rise to colonies releasing phage; the rest of the colonies were probably inhibited from reaching the critical size by competition with the mass of host in the overlay. This explanation is supported by the observation that colonies overlaid after 3 days all released phage. A similar situation appears"to exist for ØС 
that overlaid single colonies always gave rise to colony-centred plaques, whereas when washed spores were overlaid immediately about I \% of them gave plaques during the first I $4 \mathrm{~h}$ of incubation.

The unusual appearance of $\mathrm{VP}_{5}$ plaques after prolonged incubation may also be explained in terms of lysogeny. It is known, in some systems at least (Lieb, I953; Jacob \& Wollman, 1959), that the initial multiplicity of infection (m.o.i.) of a temperate phage affects the frequency of lysogenization. A low m.o.i. more often leads to the lytic response, while a high m.o.i. tends to give lysogeny. The concentration of phages in the plaque will remain low initially, giving rise to the clear (lysed) centre. The second, turbid, zone would arise as the phage concentration rose and this zone would eventually remove most of the remaining free phages. As the lysogenic host grew, it would release phages, initially in small numbers, and these would be responsible for the second lytic zone observed.

The origin from $\mathrm{VP}_{5}$ of clear-plaque mutants, still unable to lyse strains carrying the wildtype prophage, suggests that such mutants have lost the ability to establish or maintain lysogeny but still remain susceptible to the phage represser system. A similar situation was described for $\varnothing_{3}$ I by Lomovskaya et al. (1972), who also isolated mutants that may have produced a temperature-sensitive represser. Clear-plaque mutants of VP5 are currently being utilized in a search for nonsense-suppressors in the host (Najfeld, I973).

The chromosomal locus defined by segregation of phage immunity versus sensitivity in crosses of a presumed defective lysogen with sensitive strains is assumed to be the attachment site of the prophage. The map location of this attachment site is interesting, falling as it does within a long region of the linkage map devoid of normal auxotrophic, drugresistance or temperature-sensitive markers (Hopwood, I966a,b). Moreover, the attachment site of phage $\varnothing_{3} \mathrm{I}$, mapped by Lomovskaya et al. (197I), is probably also within the same segment of the map although its position relative to one auxotrophic locus, $\arg D$, at the edge of this region, has not yet been determined (Fig. 5). In the absence of a measure of the lengths of chromosomal intervals in physical terms it is still uncertain whether this 'silent region' of the linkage map represents a long segment of DNA devoid of genes accessible to study by the isolation of normal classes of mutations, or whether it represents a physically short region in which the probability of recombination/unit length is abnormally high (Hopwood, 1966a). The association of both prophages so far studied with this region of the linkage map is unlikely to be coincidental. Conceivably an increased recombination frequency/unit length of the chromosome in this region of the linkage map may occur through some kind of site-specific recombination involving prophage attachment sites.

The work reported in this paper has defined a new system involving a temperate phage and a genetically well-characterized host streptomycete, Streptomyces coelicolor A3(2). We have now available at least one virulent phage for $S$. coelicolor A3(2), VPI I (Dowding, I973), and two heteroimmune temperate phages, $\mathrm{VP}_{5}$ and $\varnothing_{3} \mathrm{I}$. Their further study is likely to increase knowledge of fundamental processes involving interactions between viruses and morphologically differentiated bacteria. Some of this knowledge may be applicable to practical problems of strain improvement in industrially important actinomycetes (Hopwood, 1972).

We are grateful to Miss V. Najfeld for information on the host-range of the phage VP5 and its clear-plaque mutants, to Mrs H. M. Wright for assistance with the mapping experiments, and to Dr N. D. Lomovskaya for kindly supplying strain $\mathrm{I}_{32} 6$ and phage $\varnothing_{3} \mathrm{I}$. J.E.D. gratefully acknowledges financial support in the form of a grant from Messrs Pfizer Ltd, Sandwich, Kent. 


\section{REFERENCES}

BradLey, D. E. (1967). Ultrastructure of bacteriophages and bacteriocins. Bacteriological Reviews $\mathbf{3}^{\mathbf{I}}$, 230-314.

Delić, V., Hopwood, D. A. \& Friend, E. J. (1970). Mutagenesis by $N$-methyl- $N^{\prime}$-nitro- $N$-nitrosoguanidine (NTG) in Streptomyces coelicolor. Mutation Research 9, 167-182.

Dowding, J. E. (1972). Studies on bacteriophages of Streptomyces coelicolor. Ph.D. Thesis, University of East Anglia, Norwich.

DowDING, J.E. (1973). Characterisation of a bacteriophage virulent for Streptomyces coelicolor A3(2). Journal of General Microbiology 76, 163-1 76.

Hopwood, D. A. (1959). Linkage and the mechanism of recombination in Streptomyces coelicolor. Annals of the New York Academy of Sciences 8r, 887-898.

Hopwood, D. A. (I966a). Nonrandom location of temperature-sensitive mutants on the linkage map of Streptomyces coelicolor. Genetics 54, I I69-i 176.

Hopwood, D. A. (1966 b). Lack of constant genome ends in Streptomyces coelicolor. Genetics 54, I I 77-I I 84.

Hopwood, D. A. (1967). Genetic analysis and genome structure in Streptomyces coelicolor. Bacteriological Reviews 3I, 373-403.

Hopwood, D. A. (1972). Genetics of the actinomycetales. In Actinomycetales: characteristics and practical importance, pp. 131-153. Edited by G. Sykes and F. A. Skinner. London: Academic Press.

Hopwood, D. A., Chater, K. F., Dowding, J. E. \& Vivian, A. (I973). Advances in Streptomyces coelicolor genetics. Bacteriological Reviews (in the Press).

Hopwood, D. A., Harold, R. J., Vivian, A. \& Ferguson, H. M. (1969). A new kind of fertility variant in Streptomyces coelicolor. Genetics 62, 46I -477 .

JaCOB, F. \& Wollman, E. L. (1956). Sur les processus de conjugaison et de recombinaison génétique chez E. coli. I. L'induction par conjugaison ou induction zygotique. Annales de l'Institut Pasteur 9r, 486-510.

JACOB, F. \& Wollman, E. L. (I959). Lysogeny. In The Viruses, vol. 2, pp. 319-35I. Edited by F. Burnet and W. M. Stanley. New York: Academic Press.

LIEB, M. (1953). The establishment of lysogeny in Escherichia coli. Journal of Bacteriology 65, 642-65I.

Lomovskaya, N. D., Emeljanova, L. K. \& Alikhanian, S. I. (I97I). The genetic location of prophage on the chromosome of Streptomyces coelicolor. Genetics 68, 34I-347.

Lomovskaya, N. D., Mkrtumian, N. M., Gostimskaya, N. L. \& Danilenko, V. N. (1972). Characterization of temperate actinophage $\emptyset_{3} \mathrm{I}$ isolated from Streptomyces coelicolor A3(2). Journal of Virology 9 , 258-262.

NAJfELd, V. (1973). A search for nonsense suppressors in Streptomyces coelicolor. M.Phil. Thesis, University of East Anglia, Norwich.

SERMONTI, G. (1969). Genetics of Antibiotic-producing Microorganisms. London: Wiley-Interscience.

ShIRLING, E. B. (1956). Studies on relationships between actinophage and variation in Streptomyces. I. Morphology of phage production and lysis in a streptomycete carrying a temperate phage. Virology 2, 272-283.

Vivian, A. (1971). Genetic control of fertility in Streptomyces coelicolor A3(2): plasmid involvement in the interconversion of UF and IF strains. Journal of General Microbiology 69, 353-364.

Vivian, A. \& Hopwoon, D. A. (1970). Genetic control of fertility in Streptomyces coelicolor A3(2): the IF fertility type. Journal of General Microbiology 64, IOI-I 17.

WELSCH, M. (1956). Evidence for the occurrence of true lysogeny among actinomycetes. Virology 2, $703-704$.

Welsch, M. (1959). Lysogenicity in streptomycetes. Annals of the New York Academy of Sciences 81, 974993.

WelsCh, M., MINON, A. \& SchönfEld, J. K. (I955). Isolation of actinophages. Experientia Ir, 24-25. 